\title{
Blow up of the solutions of the nonlinear parabolic equation
}

\author{
Svetlin G. Georgiev \\ Communicated by Y. Charles Li, received March 25, 2008.
}

\begin{abstract}
In this paper the Cauchy problem for nonlinear parabolic equation is investigated. We prove that the Cauchy problem has one nontrivial solution $u(t, r)$ in the form $u(t, r)=v(t) \omega(r) \in \mathcal{C}\left([0,1) L^{2}\left(\left[r_{0}, \infty\right)\right)\right)$ for which $\lim _{t \longrightarrow 1}\|u\|_{\left.L^{2}\left(\left[r_{0}, \infty\right)\right)\right)}=\infty$, where $r=|x|, r_{0} \geq 1$ is arbitrary chosen and fixed. Also, we prove that the solution map is not uniformly continuous.
\end{abstract}

\section{Contents}

1. Introduction

2. Proof of Main Result

3. Appendix 10

References 14

\section{Introduction}

In this paper we consider the Cauchy problem

$$
\begin{aligned}
& \text { (1.1) } u_{t}-\Delta u=f(t,|x|, u), \quad t \in[0,1], \quad|x| \geq r_{0}, \quad n \geq 2, \\
& (1.2) \quad u(0, x)=u_{0}(x) \in L^{2}\left(\mathcal{R}^{n} \backslash\left\{|x|<r_{0}\right\}\right),
\end{aligned}
$$

where $r_{0} \geq 1$ is arbitrary chosen and fixed, $f(t,|x|, u) \in \mathcal{C}^{1}([0,1]) \times \mathcal{C}^{1}\left(\left[r_{0}, \infty\right)\right) \times$ $\mathcal{C}^{1}\left(\mathcal{R}^{1}\right), a|u| \leq f_{u}^{\prime}(t,|x|, u) \leq b|u|$ for every $t \in[0,1]$, for every $|x| \geq r_{0}, a$ and $b$ are fixed positive constants, $f(t,|x|, 0)=0$ for every $t \in[0,1], \forall|x| \geq r_{0}$.

We will prove that the Cauchy problem (1.1), (1.2) has a nontrivial solution $u(t, r)$ in the form $u(t, r)=v(t) \omega(r) \in L^{2}\left(\left[r_{0}, \infty\right)\right)$ for every $t \in[0,1]$, for which $\lim _{t \longrightarrow 1}\|u\|_{L^{2}\left(\left[r_{0}, \infty\right)\right)}=\infty$. Also we will prove that the solution map is not uniformly continuous. When we say that the solution map $u_{\circ} \longrightarrow u(t, r)$ is uniformly

1991 Mathematics Subject Classification. 35.

Key words and phrases. Blow up, parabolic equation, Cauchy problem, uniformly continuous. 
continuous we mean: for every positive constant $\epsilon$ there exists positive constant $\delta$ such that for any two solutions $u, v$ of the Cauchy problem (1.1), (1.2), so that

$$
E(0, u-v) \leq \delta
$$

the following inequality holds

$$
E(t, u-v) \leq \epsilon \quad \text { for } \quad \forall t \in[0,1]
$$

where

$$
E(t, u):=\|u(t, \cdot)\|_{L^{2}\left(\left[r_{0}, \infty\right)\right)}^{2}+\left\|\frac{\partial}{\partial r} u(\cdot, r)\right\|_{L^{2}\left(\left[r_{0}, \infty\right)\right)}^{2} .
$$

Here we use the approach which is used in [1], [2], [3], [4]. In the accessible literature there are too many methods for investigations of this problem which are different than the method which we propose in this paper.

Our main result is:

Theorem 1.1. Let $n \geq 2$ is fixed, $r_{0} \geq 1$ is fixed, $f(t,|x|, u) \in \mathcal{C}^{1}([0,1]) \times$ $\mathcal{C}^{1}\left(\left[r_{0}, \infty\right)\right) \times \mathcal{C}^{1}\left(\mathcal{R}^{1}\right), a|u| \leq f_{u}^{\prime}(t,|x|, u) \leq b|u|$ for every $t \in[0,1]$, for every $|x| \geq r_{0}$, $a$ and $b$ are fixed positive constants, $f(t,|x|, 0)=0$. Then the problem of Cauchy (1.1), (1.2) has one nontrivial solution $u=u(t, r) \in \mathcal{C}\left([0,1) L^{2}\left(\left[r_{0}, \infty\right)\right)\right)$ for which $\lim _{t \longrightarrow 1}\|u\|_{L^{2}\left(\left[r_{o}, \infty\right)\right)}=\infty$. Also the solution map is not uniformly continuous.

The paper is organized as follows. In section 2 we will prove our main result. In the appendix we will prove a result which we will use for the proof of our main result.

\section{Proof of Main Result}

Here $r_{0} \geq 1$ is fixed, $n \geq 2$ is fixed.

Since we will search a positive solution $u(t, r)$ in the form $u(t, r)=v(t) \omega(r)$ we rewrite the problem (1.1), (1.2) as follows

$$
\begin{aligned}
& \text { (2.1) } u_{t}-u_{r r}-\frac{n-1}{r} u_{r}=f(t, r, u), \quad t \in[0,1], \quad r \geq r_{0}, \\
& \text { (2.2) } u(0, r)=u_{0}(r) \in L^{2}\left(\left[r_{0}, \infty\right)\right) .
\end{aligned}
$$

For fixed positive constants $n \geq 1, r_{0} \geq 1, a, b$ we suppose that the constants $A_{1}, A_{2}, A, B, c_{1}, d_{1}$ satisfy the following conditions

$$
\text { (i1) }\left\{\begin{array}{l}
1 \leq r_{0} \leq c_{1} \leq d_{1}, 0<A_{1} \leq A_{2}, 0<\frac{1}{A} \leq \frac{1}{B} \\
A_{1}>2 \\
\left(A_{1}-1\right) \frac{d_{1}^{n}}{\left(1+d_{1}\right)^{n}} \geq 1
\end{array}\right.
$$

Example. Let $n=14, r_{0} \geq 2$. Let also

$$
\begin{aligned}
& a=2 r_{0}^{11 n}, b=4 r_{0}^{11 n}, A=r_{0}^{n}, B=\frac{1}{2} r_{0}^{n}, \\
& A_{1}=r_{0}^{10 n}, A_{2}=2 r_{0}^{10 n}, c_{1}=r_{0}+1, d_{1}=r_{0}+2 .
\end{aligned}
$$

We note that $\frac{a}{2 A}=A_{1}$.

For fixed $n \geq 1, r_{0} \geq 1, a, b$ bellow we suppose that the constants $A_{1}, A_{2}, A$, $B, c_{1}, d_{1}$ satisfy the conditions ( $\left.i 1\right)$. Also we will suppose that the function $v(t)$ is fixed function which satisfies the following hypotheses

$$
\begin{aligned}
& (H 1) \quad v(t) \in \mathcal{C}^{3}([0,1]), \quad v(t)>0 \quad \forall t \in[0,1], \quad \frac{v^{\prime}(t)}{v(t)}>0 \quad \forall t \in[0,1], \\
& (H 2) \quad A_{1} \leq \frac{v^{\prime}(t)}{v(t)} \leq A_{2} \quad \forall t \in[0,1], \quad \lim _{t \longrightarrow 1}\left(\frac{v^{\prime}(t)}{v(t)}-\frac{a}{2 A}\right)=+0 .
\end{aligned}
$$


There exists a function $v(t)$ which satisfies the conditions $(H 1),(H 2)$. For instance $v(t)=e^{\frac{a}{2 A}(t-1)}$, where $a, b, A, B, A_{1}, A_{2}, c_{1}, d_{1}$ are the constants from the above example.

Let $N$ be the set

$$
\begin{aligned}
& N=\left\{u(t, r): u(t, r) \in \mathcal{C}^{1}([0,1]) \quad \forall r \geq r_{0},\right. \\
& u(t, \infty)=u_{r}(t, \infty)=0 \quad \forall t \in[0,1], \\
& r^{\alpha}\left|\partial_{r}^{\beta} u(t, r)\right| \leq 1 \quad \forall t \in[0,1], \forall r \geq r_{0}, \quad \forall \alpha \in \mathcal{N} \cup\{0\}, \beta=0,1, \\
& u(t, r) \geq 0 \quad \forall t \in[0,1], \forall r \geq r_{0}, u(t, r) \leq \frac{1}{B} \quad \forall t \in[0,1], \forall r \geq r_{0}, \\
& u(t, r) \geq \frac{1}{A} \quad \forall t \in[0,1], \forall r \in\left[c_{1}, d_{1}\right], \\
& \left.u(t, r) \in L^{2}\left(\left[r_{0}, \infty\right)\right) \quad \forall t \in[0,1]\right\} .
\end{aligned}
$$

For $u \in N$, fixed $n \geq 1$ and for every fixed $t \in[0,1]$ we define the operator

$$
P(u)=\int_{r}^{\infty} \frac{1}{s^{n}} \int_{s}^{\infty} \tau^{n}\left(\frac{v^{\prime}(t)}{v(t)} u-f(t, \tau, u)\right) d \tau d s, \quad r \geq r_{0} .
$$

We put

$$
u_{0}(r)=\int_{r}^{\infty} \frac{1}{s^{n}} \int_{s}^{\infty} \tau^{n}\left(\frac{v^{\prime}(0)}{v(0)} u_{0}-f\left(0, \tau, u_{0}\right)\right) d \tau d s, \quad r \geq r_{0}
$$

Bellow we will prove that $u_{0}(r) \in L^{2}\left(\left[r_{0}, \infty\right)\right)$ exists.

Theorem 2.1. Let $n \geq 2$ be fixed, $r_{0} \geq 1$ be fixed, the positive constants $a, b$, $a \leq b$, are fixed, $f(t,|x|, u) \in \mathcal{C}^{1}([0,1]) \times \mathcal{C}^{1}\left(\left[r_{0}, \infty\right)\right) \times \mathcal{C}^{1}\left(\mathcal{R}^{1}\right), a|u| \leq f_{u}^{\prime}(t,|x|, u) \leq$ $b|u|$ for every $t \in[0,1]$, for every $|x| \geq r_{0}, f(t,|x|, 0)=0$ for every $t \in[0,1]$ and for every $|x| \geq r_{0}$. Let also the positive constants $c_{1}, d_{1}, A_{1}, A_{2}, A, B$ are fixed which satisfy the conditions $(i 1)$, the function $v(t)$ is fixed which satisfies the hypotheses $(H 1),(H 2)$. Then the Cauchy problem (2.1), (2.2) has one unique solution $u(t, r)$ in the form $u(t, r)=v(t) \omega(r)$ for which $u(t, r) \in N, \lim _{t \longrightarrow 1}\|u\|_{L^{2}\left(\left[r_{0}, \infty\right)\right)}=\infty$.

Proof. Here and bellow we will suppose that $t \in[0,1]$ is fixed.

First we will prove that $P: N \longrightarrow N$. Let $u \in N$ is fixed.

1) Since $u(t, r) \in \mathcal{C}^{1}([0,1])$ for every $r \geq r_{0}, f(t, r, u) \in \mathcal{C}^{1}([0,1]) \times \mathcal{C}^{1}\left(\left[r_{0}, \infty\right)\right) \times$ $\mathcal{C}^{1}\left(\mathcal{R}^{1}\right), v(t) \in \mathcal{C}^{3}([0,1])$, we have $P(u) \in \mathcal{C}^{1}([0,1])$ for every $r \geq r_{0}$. Also

$$
\begin{aligned}
& P(u)_{\left.\right|_{r=\infty}}=0 \\
& \frac{\partial P(u)}{\partial r}=-\frac{1}{r^{n}} \int_{r}^{\infty} \tau^{n}\left(\frac{v^{\prime}(t)}{v(t)} u-f(t, \tau, u)\right) d \tau \\
& \left.\frac{\partial P(u)}{\partial r}\right|_{r=\infty}=0 .
\end{aligned}
$$

We note that from the conditions of the Theorem 2.1 we have $f_{u}^{\prime}(t, \tau, u) \leq b u$. From here and from $f(t, \tau, 0)=0$ we get $f(t, \tau, u) \leq \frac{b}{2} u^{2}$. From the defintion of the set $N$ we have $u \leq \frac{1}{B}$. Therefore $f(t, \tau, u) \leq \frac{b}{2 B} u$ for every $t \in[0,1], \tau \geq r_{0}$. Also we have $f(t, r, u) \geq \frac{a}{2} u^{2}$ for every $t \in[0,1]$ and for every $r \geq r_{0}$.

Let $\alpha \in \mathcal{N} \cup\{0\}$ is arbitrary chosen and fixed, $k \in \mathcal{N}$ is enough large such that

$$
\begin{aligned}
& k>\alpha+3 \\
& A_{2}+\frac{b}{2 B} \leq k-1
\end{aligned}
$$


Then for $r \geq r_{0}$ we have

$$
\begin{aligned}
& \left|r^{\alpha} P(u)\right|=\left|r^{\alpha} \int_{r}^{\infty} \frac{1}{s^{n}} \int_{s}^{\infty} \tau^{n}\left(\frac{v^{\prime}(t)}{v(t)} u-f(t, \tau, u)\right) d \tau d s\right| \leq \\
& \leq r^{\alpha} \int_{r}^{\infty} \frac{1}{s^{n}} \int_{s}^{\infty} \tau^{n}\left(\frac{v^{\prime}(t)}{v(t)} u+f(t, \tau, u)\right) d \tau d s \leq \\
& \text { here we use that } f(t, \tau, u) \leq \frac{b}{2 B} u \\
& \leq r^{\alpha} \int_{r}^{\infty} \frac{1}{s^{n}} \int_{s}^{\infty} \tau^{n}\left(\frac{v^{\prime}(t)}{v(t)} u+\frac{b}{2 B} u\right) d \tau d s \leq \\
& \leq r^{\alpha} \int_{r}^{\infty} \frac{1}{s^{n}} \int_{s}^{\infty} \tau^{n}\left(A_{2} u+\frac{b}{2 B} u\right) d \tau d s= \\
& =r^{\alpha} \int_{r}^{\infty} \frac{1}{s^{n}} \int_{s}^{\infty} \tau^{n}\left(A_{2}+\frac{b}{2 B}\right) u d \tau d s= \\
& =\left(A_{2}+\frac{b}{2 B}\right) r^{\alpha} \int_{r}^{\infty} \frac{1}{s^{n}} \int_{s}^{\infty} \tau^{n} u d \tau d s= \\
& =\left(A_{2}+\frac{b}{2 B}\right) r^{\alpha} \int_{r}^{\infty} \frac{1}{s^{n}} \int_{s}^{\infty} \frac{\tau^{k+n} u}{\tau^{k}} d \tau d s \leq
\end{aligned}
$$

here we use that from the definition of the set $N$ we have $\tau^{k+n} u \leq 1$

$$
\begin{aligned}
& \leq\left(A_{2}+\frac{b}{2 B}\right) r^{\alpha} \int_{r}^{\infty} \frac{1}{s^{n}} \int_{s}^{\infty} \frac{1}{\tau^{k}} d \tau d s= \\
& =\frac{1}{(k-1)(k+n-2)}\left(A_{2}+\frac{b}{2 B}\right) \frac{1}{r^{k+n-\alpha-2}} \leq \\
& \leq \frac{1}{(k-1)(k+n-2)}\left(A_{2}+\frac{b}{2 B}\right) \frac{1}{r_{0}^{k+n-\alpha-2}} \leq 1 .
\end{aligned}
$$

In the last inequality we use our choice of the constant $k$.

Let $k$ is the same as above. Then for $r \geq r_{0}$ we have

$$
\begin{aligned}
& \left|r^{\alpha} \frac{\partial P(u)}{\partial r}\right|=\left|r^{\alpha} \frac{1}{r^{n}} \int_{r}^{\infty} \tau^{n}\left(\frac{v^{\prime}(t)}{v(t)} u-f(t, \tau, u)\right) d \tau\right| \leq \\
& \leq r^{\alpha} \frac{1}{r^{n}} \int_{r}^{\infty} \tau^{n}\left(\frac{v^{\prime}(t)}{v(t)} u+f(t, \tau, u)\right) d \tau \leq \\
& \text { here we use thatf }(t, \tau, u) \leq \frac{b}{2 B} u \\
& \leq r^{\alpha} \frac{1}{r^{n}} \int_{r}^{\infty} \tau^{n}\left(\frac{v^{\prime}(t)}{v(t)} u+\frac{b}{2 B} u\right) d \tau \leq \\
& \leq r^{\alpha} \frac{1}{r^{n}} \int_{r}^{\infty} \tau^{n}\left(A_{2} u+\frac{b}{2 B} u\right) d \tau= \\
& =r^{\alpha} \frac{1}{r^{n}} \int_{r}^{\infty} \tau^{n}\left(A_{2}+\frac{b}{2 B}\right) u d \tau= \\
& =\left(A_{2}+\frac{b}{2 B}\right) r^{\alpha} \frac{1}{r^{n}} \int_{r}^{\infty} \frac{\tau^{k+n} u}{\tau^{k}} d \tau \leq
\end{aligned}
$$

here we use that from the definition of the set $N$ we have $\tau^{k+n} u \leq 1$

$$
\begin{aligned}
& \leq\left(A_{2}+\frac{b}{2 B}\right) r^{\alpha} \frac{1}{r^{n}} \int_{r}^{\infty} \frac{1}{\tau^{k}} d \tau= \\
& =\left(A_{2}+\frac{b}{2 B}\right) \frac{1}{k-1} \frac{1}{r^{n+k-\alpha-1}} \leq \\
& \leq\left(A_{2}+\frac{b}{2 B}\right) \frac{1}{k-1} \frac{1}{r_{0}^{n+k-\alpha-1}} \leq 1 .
\end{aligned}
$$

In the last inequality we use our choice of the constant $k$.

2) Now we will prove that for every fixed $t \in[0,1]$ and for every $r \geq r_{0}$ we have $P(u) \geq 0$.

Really, for $k \in \mathcal{N}$ for which $\frac{b}{r_{0}^{n k}}<1$ we have

$$
P(u)=\int_{r}^{\infty} \frac{1}{s^{n}} \int_{s}^{\infty} \tau^{n}\left(\frac{v^{\prime}(t)}{v(t)} u-f(t, \tau, u)\right) d \tau d s=
$$

now we apply the middle point theorem

$$
=\int_{r}^{\infty} \frac{1}{s^{n}} \int_{s}^{\infty} \tau^{n}\left(\frac{v^{\prime}(t)}{v(t)} u-f_{u}^{\prime}(t, \tau, \xi) u\right) d \tau d s \geq
$$


here we use that $f_{u}^{\prime}(t, \tau, \xi) \leq b \xi \leq b u$

$$
\begin{aligned}
& \geq \int_{r}^{\infty} \frac{1}{s^{n}} \int_{s}^{\infty} \tau^{n}\left(\frac{v^{\prime}(t)}{v(t)}-b u\right) u d \tau d s= \\
& =\int_{r}^{\infty} \frac{1}{s^{n}} \int_{s}^{\infty} \tau^{n}\left(\frac{v^{\prime}(t)}{v(t)}-b \frac{\tau^{n k} u}{\tau^{n k}}\right) u d \tau d s \geq \\
& \text { here we use } \tau^{n k} u \leq 1 \\
& \geq \int_{r}^{\infty} \frac{1}{s^{n}} \int_{s}^{\infty} \tau^{n}\left(A_{1}-\frac{b}{r_{0}^{n k}}\right) u d \tau d s,
\end{aligned}
$$

i.e.

$$
P(u) \geq \int_{r}^{\infty} \frac{1}{s^{n}} \int_{s}^{\infty} \tau^{n}\left(A_{1}-\frac{b}{r_{0}^{n k}}\right) u d \tau d s .
$$

Since $u(t, r) \geq 0$ for every fixed $t \in[0,1]$ and for every $r \geq r_{0}$ and from our choice of the constant $k$ we have that $P(u) \geq 0$ for every $t \in[0, \overline{1}]$ and for every $r \geq r_{0}$.

3) Now we will see that for every fixed $t \in[0,1]$ and for every $r \in\left[c_{1}, d_{1}\right]$ we have $P(u) \geq \frac{1}{A}$. We suppose that $k$ is same as in $\left.\mathbf{2}\right)$. Let

$$
g(u)=\int_{r}^{\infty} \frac{1}{s^{n}} \int_{s}^{\infty} \tau^{n}\left(A_{1}-\frac{b}{r_{0}^{n k}}\right) u d \tau d s .
$$

Then

$$
g^{\prime}(u)=\int_{r}^{\infty} \frac{1}{s^{n}} \int_{s}^{\infty} \tau^{n}\left(A_{1}-\frac{b}{r_{0}^{n k}}\right) d \tau d s \geq 0 .
$$

In the last inequality we use our choice of the constant $k$. Consequently $g(u)$ is increase function of $u$. Since for every fixed $t \in[0,1]$ and for every $r \in\left[c_{1}, d_{1}\right]$ we have $u \geq \frac{1}{A}$ we get

$$
\begin{aligned}
& g(u) \geq g\left(\frac{1}{A}\right)= \\
& =g(u)=\int_{r}^{\infty} \frac{1}{s^{n}} \int_{s}^{\infty} \tau^{n}\left(A_{1}-\frac{b}{r_{0}^{n k}}\right) \frac{1}{A} d \tau d s \geq \\
& \geq \int_{d_{1}}^{d_{1}+1} \frac{1}{s^{n}} \int_{d_{1}}^{d_{1}+1} \tau^{n}\left(A_{1}-1\right) \frac{1}{A} d \tau d s \geq \\
& \geq\left(A_{1}-1\right) \frac{d_{1}^{n}}{\left(d_{1}+1\right)^{n}} \frac{1}{A} \geq \frac{1}{A} .
\end{aligned}
$$

In the last inequality we use the conditions $(i 1)$. From here and from $\left(2.2^{\prime}\right)$ we get that $P(u) \geq \frac{1}{A}$ for every fixed $t \in[0,1]$ and for every $r \in\left[c_{1}, d_{1}\right]$.

4) Now we will prove that for every fixed $t \in[0,1]$ and for every $r \geq r_{0}$ we have $P(u) \leq \frac{1}{B}$. Let $k \in \mathcal{N}$ is chosen such that

$$
\left(A_{2}+\frac{b}{2 B}\right) \frac{1}{(k-1)(n+k-2) r_{0}^{n+k-2}} \leq \frac{1}{B}, \quad k \geq 2 .
$$

Then

$$
\begin{aligned}
& P(u)=\int_{r}^{\infty} \frac{1}{s^{n}} \int_{s}^{\infty} \tau^{n}\left(\frac{v^{\prime}(t)}{v(t)} u-f(t, \tau, u)\right) d \tau d s \leq \\
& \leq \int_{r}^{\infty} \frac{1}{s^{n}} \int_{s}^{\infty} \tau^{n}\left(A_{2} u+f(t, \tau, u)\right) d \tau d s \leq \\
& \leq \int_{r}^{\infty} \frac{1}{s^{n}} \int_{s}^{\infty} \tau^{n}\left(A_{2} u+\frac{b}{2 B} u\right) d \tau d s= \\
& =\int_{r}^{\infty} \frac{1}{s^{n}} \int_{s}^{\infty}\left(A_{2}+\frac{b}{2 B}\right) \frac{\tau^{n+k} u}{\tau^{k}} d \tau d s \leq \\
& \text { here we use } \tau^{n+k} u \leq 1 \\
& \int_{r}^{\infty} \frac{1}{s^{n}} \int_{s}^{\infty}\left(A_{2}+\frac{b}{2 B}\right) \frac{1}{\tau^{k}} d \tau d s \leq \\
& \leq\left(A_{2}+\frac{b}{2 B}\right) \frac{1}{(k-1)(n+k-2) r_{0}^{n+k-2}} \leq \frac{1}{B} .
\end{aligned}
$$

In the last inequality we use our choice of the constant $k$. 
5) Now we will prove that $P(u) \in L^{2}\left(\left[r_{0}, \infty\right)\right)$ for every fixed $t \in[0,1]$. We choose $k \in \mathcal{N}$ such that $n+k-4>0$.

$$
\begin{aligned}
& \|P(u)\|_{L^{2}\left(\left[r_{0}, \infty\right)\right)}^{2}=\int_{r_{0}}^{\infty}\left(\int_{r}^{\infty} \frac{1}{s^{n}} \int_{s}^{\infty} \tau^{n}\left(\frac{v^{\prime}(t)}{v(t)} u-f(t, \tau, u)\right) d \tau d s\right)^{2} d r \leq \\
& \leq \int_{r_{0}}^{\infty}\left(\int_{r}^{\infty} \frac{1}{s^{n}} \int_{s}^{\infty} \tau^{n}\left(A_{2}+\frac{b}{2 B}\right) u d \tau d s\right)^{2} d r= \\
& =\left(A_{2}+\frac{b}{2 B}\right)^{2} \int_{r_{0}}^{\infty}\left(\int_{r}^{\infty} \frac{1}{s^{n}} \int_{s}^{\infty} \sqrt{\tau^{2 k+2 n} u} \sqrt{u} \frac{1}{\tau^{k}} d \tau d s\right)^{2} d r \leq \\
& \left(A_{2}+\frac{b}{2 B}\right)^{2} \int_{r_{0}}^{\infty}\left(\int_{r}^{\infty} \frac{1}{s^{n}} \int_{s}^{\infty} \sqrt{u} \frac{1}{\tau^{k}} d \tau d s\right)^{2} d r \leq \\
& \text { now we use the Hölder's inequality } \\
& \leq\left(A_{2}+\frac{b}{2 B}\right)^{2} \int_{r_{0}}^{\infty}\left(\int_{r}^{\infty} \frac{1}{s^{n}}\left(\int_{s}^{\infty} \frac{1}{\tau^{\frac{4 k}{3}}} d \tau\right)^{\frac{3}{4}}\left(\int_{s}^{\infty} u^{2} d \tau\right)^{\frac{1}{4}} d s\right)^{2} d r \leq \\
& \leq\left(A_{2}+\frac{b}{2 B}\right)^{2} \frac{1}{\left(\frac{4}{3} k-1\right)^{\frac{3}{2}}} \cdot \frac{1}{\left(n+k-\frac{7}{4}\right)^{2}} \frac{1}{2 n+2 k-\frac{9}{2}} \frac{1}{r_{0}^{2 n+2 k-\frac{9}{2}}}\|u\|_{L^{2}\left(\left[r_{0}, \infty\right)\right)<\infty}
\end{aligned}
$$

From 1), 2), 3), 4), 5) we conclude that $P: N \longrightarrow N$ for every fixed $t \in[0,1]$.

Now we will prove that the operator $P$ has one unique nontrivial fixed point in the set $N$.

Let $t \in[0,1)$ is fixed. Let also $u_{1} \in N, u_{2} \in N$ are fixed and $\alpha=\| u_{1}-$ $u_{2} \|_{L^{2}\left(\left[r_{0}, \infty\right)\right)} \neq 0$.

We choose $k \in \mathcal{N}, k>3$ enough large so that

$$
\frac{1}{\alpha} Q_{1}=\frac{2\left(A_{2}+\frac{b}{B}\right)^{2}}{\alpha\left(\frac{4}{3} k-1\right)^{\frac{3}{2}}\left(k+n-\frac{7}{4}\right)^{2}\left(2 n+2 k-\frac{9}{2}\right) r_{0}^{2 n+2 k-\frac{9}{2}}}<1, \quad Q_{1}<1 .
$$

Then

$$
\begin{aligned}
& \left\|P\left(u_{1}\right)-P\left(u_{2}\right)\right\|_{L^{2}\left(\left[r_{0}, \infty\right)\right)}^{2}= \\
& =\int_{r_{0}}^{\infty}\left(\int_{r}^{\infty} \frac{1}{s^{n}} \int_{s}^{\infty} \tau^{n}\left(\frac{v^{\prime}(t)}{v(t)}\left(u_{1}-u_{2}\right)-\left(f\left(t, \tau, u_{1}\right)-f\left(t, \tau, u_{2}\right)\right)\right) d \tau d s\right)^{2} d r \leq \\
& \leq \int_{r_{0}}^{\infty}\left(\int_{r}^{\infty} \frac{1}{s^{n}} \int_{s}^{\infty} \tau^{n}\left(\frac{v^{\prime}(t)}{v(t)}\left|u_{1}-u_{2}\right|+\left|f\left(t, \tau, u_{1}\right)-f\left(t, \tau, u_{2}\right)\right|\right) d \tau d s\right)^{2} d r \leq \\
& \leq \int_{r_{0}}^{\infty}\left(\int_{r}^{\infty} \frac{1}{s^{n}} \int_{s}^{\infty} \tau^{n}\left(A_{2}\left|u_{1}-u_{2}\right|+\left|f\left(t, \tau, u_{1}\right)-f\left(t, \tau, u_{2}\right)\right|\right) d \tau d s\right)^{2} d r \leq
\end{aligned}
$$

now we use the middle point theorem

$$
\begin{aligned}
& \leq \int_{r_{0}}^{\infty}\left(\int_{r}^{\infty} \frac{1}{s^{n}} \int_{s}^{\infty} \tau^{n}\left(A_{2}\left|u_{1}-u_{2}\right|+\left|f_{u}^{\prime}(t, \tau, \xi)\right|\left|u_{1}-u_{2}\right|\right) d \tau d s\right)^{2} d r \leq \\
& \leq \int_{r_{0}}^{\infty}\left(\int_{r}^{\infty} \frac{1}{s^{n}} \int_{s}^{\infty} \tau^{n}\left(A_{2}\left|u_{1}-u_{2}\right|+\frac{b}{B}\left|u_{1}-u_{2}\right|\right) d \tau d s\right)^{2} d r= \\
& =\left(A_{2}+\frac{b}{B}\right)^{2} \int_{r_{0}}^{\infty}\left(\int_{r}^{\infty} \frac{1}{s^{n}} \int_{s}^{\infty} \tau^{n}\left|u_{1}-u_{2}\right| d \tau d s\right)^{2} d r= \\
& =\left(A_{2}+\frac{b}{B}\right)^{2} \int_{r_{0}}^{\infty}\left(\int_{r}^{\infty} \frac{1}{s^{n}} \int_{s}^{\infty} \sqrt{\tau^{2 k+2 n}\left|u_{1}-u_{2}\right|} \frac{1}{\tau^{k}} \sqrt{\left|u_{1}-u_{2}\right|} d \tau d s\right)^{2} d r \leq \\
& \text { here we use that } \sqrt{\tau^{2 k+2 n}\left|u_{1}-u_{2}\right|} \leq \sqrt{2} \\
& \leq 2\left(A_{2}+\frac{b}{B}\right)^{2} \int_{r_{0}}^{\infty}\left(\int_{r}^{\infty} \frac{1}{s^{n}} \int_{s}^{\infty} \frac{1}{\tau^{k}} \sqrt{\left|u_{1}-u_{2}\right|} d \tau d s\right)^{2} d r \leq \\
& \text { here we use the Hölder } \text { inequality }^{2} \text { ine } \\
& \leq 2\left(A_{2}+\frac{b}{B}\right)^{2} \int_{r_{0}}^{\infty}\left(\int_{r}^{\infty} \frac{1}{s^{n}}\left(\int_{s}^{\infty} \frac{1}{\tau^{\frac{4 k}{3}}} d \tau\right)^{\frac{3}{4}}\left(\int_{s}^{\infty}\left|u_{1}-u_{2}\right|^{2} d \tau\right)^{\frac{1}{4}} d s\right)^{2} d r \leq \\
& \leq Q_{1}|| u_{1}-u_{2} \|_{L^{2}([0, \infty))}
\end{aligned}
$$

i.e.

$$
\left\|P\left(u_{1}\right)-P\left(u_{2}\right)\right\|_{L^{2}\left(\left[r_{0}, \infty\right)\right)}^{2} \leq Q_{1}\left\|u_{1}-u_{2}\right\|_{L^{2}\left(\left[r_{0}, \infty\right)\right)} .
$$


Since we choose the constant $k$ so that $\frac{1}{\alpha} Q_{1}<1$ we have

$$
\left\|P\left(u_{1}\right)-P\left(u_{2}\right)\right\|_{L^{2}\left(\left[r_{0}, \infty\right)\right)}^{2} \leq \frac{Q_{1}}{\alpha} \alpha\left\|u_{1}-u_{2}\right\|_{L^{2}\left(\left[r_{0}, \infty\right)\right)}^{2} \leq \frac{Q_{1}}{\alpha}\left\|u_{1}-u_{2}\right\|_{L^{2}\left(\left[r_{0}, \infty\right)\right)}^{2} .
$$

From here and from the following theorem

Theorem[5, p. 294] Let $B$ be the completely metric space for which $A B \subset B$ and for the operator $A$ is hold the following condition

$$
\rho(A x, A y) \leq L(\alpha, \beta) \rho(x, y), \quad x, y \in B, \alpha \leq \rho(x, y) \leq \beta,
$$

where $L(\alpha, \beta)<1$ for $0<\alpha \leq \beta<\infty$. Then the operator $A$ has exactly one fixed point in the space $B$.

we conclude that the operator $P$ has one unique fixed point $u$ in the set $N$. We note that the set $N$ is a closed subset of the space $L^{2}\left(\left[r_{0}, \infty\right)\right)$ for every fixed $t \in[0,1]$ (see lemma 3.1 in the appendix of this paper) As in the proof of the Proposition $2.1,2.2$ [4] we have that the fixed point $u$ satisfies the equation $(2.1)$ with initial data

$$
u_{0}=\int_{r}^{\infty} \frac{1}{s^{n}} \int_{s}^{\infty} \tau^{n}\left(\frac{v^{\prime}(0)}{v(0)} u_{0}-f\left(0, \tau, u_{0}\right)\right) d \tau d s, r \geq r_{0}
$$

We have that $u_{0} \in L^{2}\left(\left[r_{0}, \infty\right)\right)$.

Now we will prove that

$$
\lim _{t \longrightarrow 1}\|u\|_{L^{2}\left(\left[r_{0}, \infty\right)\right)}=\infty .
$$

For $k \in \mathcal{N}$ we put

$$
\begin{aligned}
Q_{2} & =\left(A_{2}+\frac{b}{2 B}\right)^{2} \frac{1}{\left(\frac{4}{3} k-1\right)^{\frac{3}{2}}\left(n+k-\frac{7}{4}\right)^{2}\left(2 n+2 k-\frac{9}{2}\right) r_{0}^{2 n+2 k-\frac{9}{2}}} \\
Q_{3} & =2 A^{2}\left(\frac{v^{\prime}(t)}{v(t)}-\frac{a}{2 A}\right)^{2} d_{1}^{2 n}\left(d_{1}-c_{1}\right)^{3} \frac{1}{c_{1}^{2 n}} \\
Q_{4} & =\left(A_{1}-\frac{b}{2 B}\right) \frac{1}{A} \frac{c_{1}^{n}}{d_{1}^{n}}\left(d_{1}-c_{1}\right)^{\frac{5}{2}} .
\end{aligned}
$$

We choose the constant $k \in \mathcal{N}$ such that

$$
1-10 \frac{Q_{2}}{Q_{4}}>0 \text {. }
$$

Then

$$
\begin{aligned}
& \|u\|_{L^{2}\left(\left[r_{0}, \infty\right)\right)}^{2}=\int_{r_{0}}^{\infty}\left(\int_{r}^{\infty} \frac{1}{s^{n}} \int_{s}^{\infty} \tau^{n}\left(\frac{v^{\prime}(t)}{v(t)} u-f(t, \tau, u)\right) d \tau d s\right)^{2} d r= \\
& =\int_{r_{0}}^{c_{1}}\left(\int_{r}^{\infty} \frac{1}{s^{n}} \int_{s}^{\infty} \tau^{n}\left(\frac{v^{\prime}(t)}{v(t)} u-f(t, \tau, u)\right) d \tau d s\right)^{2} d r+ \\
& +\int_{c_{1}}^{\infty}\left(\int_{r}^{\infty} \frac{1}{s^{n}} \int_{s}^{\infty} \tau^{n}\left(\frac{v^{\prime}(t)}{v(t)} u-f(t, \tau, u)\right) d \tau d s\right)^{2} d r .
\end{aligned}
$$

Let

$$
\begin{aligned}
J_{1} & :=\int_{r_{0}}^{c_{1}}\left(\int_{r}^{\infty} \frac{1}{s^{n}} \int_{s}^{\infty} \tau^{n}\left(\frac{v^{\prime}(t)}{v(t)} u-f(t, \tau, u)\right) d \tau d s\right)^{2} d r \\
J_{2} & :=\int_{c_{1}}^{\infty}\left(\int_{r}^{\infty} \frac{1}{s^{n}} \int_{s}^{\infty} \tau^{n}\left(\frac{v^{\prime}(t)}{v(t)} u-f(t, \tau, u)\right) d \tau d s\right)^{2} d r
\end{aligned}
$$

Then

$$
\|u\|_{L^{2}\left(\left[r_{0}, \infty\right)\right)}^{2}=J_{1}+J_{2}
$$


For $J_{1}$ we have the following estimate

$$
\begin{aligned}
& J_{1} \leq \int_{r_{0}}^{\infty}\left(\int_{r}^{\infty} \frac{1}{s^{n}} \int_{s}^{\infty} \tau^{n}\left(\frac{v^{\prime}(t)}{v(t)} u+f(t, \tau, u)\right) d \tau d s\right)^{2} d r \leq \\
& \leq \int_{r_{0}}^{\infty}\left(\int_{r}^{\infty} \frac{1}{s^{n}} \int_{s}^{\infty} \tau^{n}\left(A_{2} u+\frac{b}{2 B} u\right) d \tau d s\right)^{2} d r= \\
& =\left(A_{2}+\frac{b}{2 B}\right)^{2} \int_{r_{0}}^{\infty}\left(\int_{r}^{\infty} \frac{1}{s^{n}} \int_{s}^{\infty} \sqrt{\tau^{2 k+2 n} u} \frac{1}{\tau^{k}} \sqrt{u} d \tau d s\right)^{2} d r \leq \\
& \text { here we use that } \sqrt{\tau^{2 k+2 n} u} \leq 1 \\
& \leq\left(A_{2}+\frac{b}{2 B}\right)^{2} \int_{r_{0}}^{\infty}\left(\int_{r}^{\infty} \frac{1}{s^{n}} \int_{s}^{\infty} \frac{1}{\tau^{k}} \sqrt{u} d \tau d s\right)^{2} d r \leq \\
& \text { now use the Hölder's inequlity } \\
& \leq\left(A_{2}+\frac{b}{2 B}\right)^{2} \int_{r_{0}}^{\infty}\left(\int_{r}^{\infty} \frac{1}{s^{n}}\left(\int_{s}^{\infty} \frac{1}{\tau^{\frac{4 k}{3}}} d \tau\right)^{\frac{3}{4}}\left(\int_{s}^{\infty} u^{2} d \tau\right)^{\frac{1}{4}} d s\right)^{2} d r \leq \\
& \leq Q_{2}\|u\|_{L^{2}\left(\left[r_{0}, \infty\right)\right)} .
\end{aligned}
$$

$$
J_{1} \leq Q_{2}\|\tilde{u}\|_{L^{2}\left(\left[r_{0}, \infty\right)\right)} .
$$

Now we consider $J_{2}$. For it we have

$$
\begin{aligned}
& J_{2}=\int_{c_{1}}^{\infty}\left(\int_{r}^{\infty} \frac{1}{s^{n}} \int_{s}^{\infty} \tau^{n}\left(\frac{v^{\prime \prime}(t)}{v(t)} u-f(t, \tau, u)\right) d \tau d s\right)^{2} d r= \\
& \int_{c_{1}}^{d_{1}}\left(\int_{c_{1}}^{d_{1}} \frac{1}{s^{n}} \int_{s}^{\infty} \tau^{n}\left(\frac{v^{\prime}(t)}{v(t)} u-f(t, \tau, u)\right) d \tau d s+\right. \\
& \left.\int_{d_{1}}^{\infty} \frac{1}{s^{n}} \int_{s}^{\infty} \tau^{n}\left(\frac{v^{\prime}(t)}{v(t)} u-f(t, \tau, u)\right) d \tau d s\right)^{2} d r+ \\
& +\int_{d_{1}}^{\infty}\left(\int_{r}^{\infty} \frac{1}{s^{n}} \int_{s}^{\infty} \tau^{n}\left(\frac{v^{\prime}(t)}{v(t)} u-f(t, \tau, u)\right) d \tau d s\right)^{2} d r= \\
& =\int_{c_{1}}^{d_{1}}\left(\int_{c_{1}}^{d_{1}} \frac{1}{s^{n}} \int_{c_{1}}^{d_{1}} \tau^{n}\left(\frac{v^{\prime}(t)}{v(t)} u-f(t, \tau, u)\right) d \tau d s+\right. \\
& \int_{c_{1}}^{d_{1}} \frac{1}{s^{n}} \int_{d_{1}}^{\infty} \tau^{n}\left(\frac{v^{\prime}(t)}{v(t)} u-f(t, \tau, u)\right) d \tau d s+ \\
& \left.+\int_{d_{1}}^{\infty} \frac{1}{s^{n}} \int_{s}^{\infty} \tau^{n}\left(\frac{v^{\prime}(t)}{v(t)} u-f(t, \tau, u)\right) d \tau d s\right)^{2} d r+ \\
& +\int_{d_{1}}^{\infty}\left(\int_{r}^{\infty} \frac{1}{s^{n}} \int_{s}^{\infty} \tau^{n}\left(\frac{v^{\prime}(t)}{v(t)} u-f(t, \tau, u)\right) d \tau d s\right)^{2} d r \leq \\
& \text { here we use the inequality }(a+b)^{2} \leq 2\left(a^{2}+b^{2}\right) \\
& \leq 4 \int_{c_{1}}^{d_{1}}\left(\int_{c_{1}}^{d_{1}} \frac{1}{s^{n}} \int_{d_{1}}^{\infty} \tau^{n}\left(\frac{v^{\prime}(t)}{v(t)} u-f(t, \tau, u)\right) d \tau d s\right)_{2}^{2} d r+ \\
& +4 \int_{c_{1}}^{d_{1}}\left(\int_{d_{1}}^{\infty} \frac{1}{s^{n}} \int_{s}^{\infty} \tau^{n}\left(\frac{v^{\prime}(t)}{v(t)} u-f(t, \tau, u)\right) d \tau d s\right)^{2} d r+ \\
& +2 \int_{c_{1}}^{d_{1}}\left(\int_{c_{1}}^{d_{1}} \frac{1}{s^{n}} \int_{c_{1}}^{d_{1}} \tau^{n}\left(\frac{v^{\prime \prime}(t)}{v(t)} \tilde{u}-f(\tilde{u})\right) d \tau d s\right)^{2} d r+ \\
& +\int_{d_{1}}^{\infty}\left(\int_{r}^{\infty} \frac{1}{s^{n}} \int_{s}^{\infty} \tau^{n}\left(\frac{v^{\prime}(t)}{v(t)} u-f(t, \tau, u)\right) d \tau d s\right)^{2} d r \leq \\
& 2 \int_{c_{1}}^{d_{1}}\left(\int_{c_{1}}^{d_{1}} \frac{1}{s^{n}} \int_{c_{1}}^{d_{1}} \tau^{n}\left(\frac{v^{\prime}(t)}{v(t)} u-f(t, \tau, u)\right) d \tau d s\right)^{2} d r+9 Q_{2}\|u\|_{L^{2}\left(\left[r_{0}, \infty\right)\right)} \leq \\
& \text { here we use that } u \geq \frac{1}{A} \\
& \leq 9 Q_{2}\|\tilde{u}\|_{L^{2}\left(\left[r_{0}, \infty\right)\right)}+2 \frac{d_{1}^{2 n}}{c_{1}^{2 n}}\left(\frac{v^{\prime}(t)}{v(t)}-\frac{a}{2 A}\right)^{2}\left(d_{1}-c_{1}\right)^{3} A^{2}\left(\int_{c_{1}}^{d_{1}} u^{2} d \tau\right)^{2} \leq \\
& \leq 9 Q_{2}\|\tilde{u}\|_{L^{2}\left(\left[r_{0}, \infty\right)\right)}+2 \frac{d_{1}^{2 n}}{c_{1}^{2 n}}\left(\frac{v^{\prime}(t)}{v(t)}-\frac{a}{2 A}\right)^{2}\left(d_{1}-c_{1}\right)^{3} A^{2}\|u\|_{L^{2}\left(\left[r_{0}, \infty\right)\right)}^{4} .
\end{aligned}
$$

Then

$$
J_{2} \leq 9 Q_{2}\|u\|_{L^{2}\left(\left[r_{0}, \infty\right)\right)}+Q_{3}\|u\|_{L^{2}\left(\left[r_{0}, \infty\right)\right)}^{4} .
$$

From here and from $(2.3),(2.4)$ we get

$$
\|u\|_{L^{2}\left(\left[r_{0}, \infty\right)\right)}^{2} \leq 10 Q_{2}\|u\|_{L^{2}\left(\left[r_{0}, \infty\right)\right)}+Q_{3}\|u\|_{L^{2}\left(\left[r_{0}, \infty\right)\right)}^{4} .
$$


Also we note

$$
\begin{aligned}
& \|u\|_{L^{2}\left(\left[r_{0}, \infty\right)\right)}=\left(\int_{r_{0}}^{\infty}\left(\int_{r}^{\infty} \frac{1}{s^{n}} \int_{s}^{\infty} \tau^{n}\left(\frac{v^{\prime}(t)}{v(t)} u-f(t, \tau, u)\right) d \tau d s\right)^{2} d r\right)^{\frac{1}{2}} \geq \\
& \geq\left(\int_{c_{1}}^{d_{1}}\left(\int_{c_{1}}^{d_{1}} \frac{1}{s^{n}} \int_{c_{1}}^{d_{1}} \tau^{n}\left(\frac{v^{\prime}(t)}{v(t)} u-f(t, \tau, u)\right) d \tau d s\right)^{2} d r\right)^{\frac{1}{2}} \geq \\
& \geq\left(\int_{c_{1}}^{d_{1}}\left(\int_{c_{1}}^{d_{1}} \frac{1}{s^{n}} \int_{c_{1}}^{d_{1}} \tau^{n}\left(A_{1}-\frac{b}{2 B}\right) \frac{1}{A} d \tau d s\right)^{2} d r\right)^{\frac{1}{2}} \geq \\
& \geq \frac{c_{1}^{n}}{d_{1}^{n}}\left(A_{1}-\frac{b}{2 B}\right) \frac{1}{A}\left(d_{1}-c_{1}\right)^{\frac{5}{2}} .
\end{aligned}
$$

From here and from (2.5) we get

$$
Q_{4}\|u\|_{L^{2}\left(\left[r_{0}, \infty\right)\right)} \leq\|u\|_{L^{2}\left(\left[r_{0}, \infty\right)\right)}^{2} \leq 10 Q_{2}\|u\|_{L^{2}\left(\left[r_{0}, \infty\right)\right)}+Q_{3}\|u\|_{L^{2}\left(\left[r_{0}, \infty\right)\right)}^{4}
$$

Then

$$
\left(Q_{4}-10 Q_{2}\right)\|u\|_{L^{2}\left(\left[r_{0}, \infty\right)\right)} \leq Q_{3}\|u\|_{L^{2}\left(\left[r_{0}, \infty\right)\right)}^{4}
$$

From our choice of the constant $k$ we have that $Q_{4}-10 Q_{2}>0$. Therefore

$$
\frac{\left(Q_{4}-10 Q_{2}\right)}{Q_{3}} \leq\|u\|_{L^{2}\left(\left[r_{0}, \infty\right)\right)}^{3}
$$

from where

$$
\lim _{t \rightarrow 1}\|u\|_{L^{2}\left(\left[r_{0}, \infty\right)\right)}=\infty
$$

because $\lim _{t \longrightarrow 1} Q_{3}=0$ (see $(H 2)$ ).

Theorem 2.2. Let $n \geq 2$ be fixed, $r_{0} \geq 1$ be fixed, the positive constants $a, b$, $a \leq b$, are fixed, $f(t,|x|, u) \in \mathcal{C}^{1}([0,1]) \times \mathcal{C}^{1}\left(\left[r_{0}, \infty\right)\right) \times \mathcal{C}^{1}\left(\mathcal{R}^{1}\right), a|u| \leq f_{u}^{\prime}(t,|x|, u) \leq$ $b|u|$ for every $t \in[0,1]$, for every $|x| \geq r_{0}, f(t,|x|, 0)=0$. Let also the positive constants $c_{1}, d_{1}, A_{1}, A_{2}, A, B$ are fixed which satisfy the conditions $(i 1)$, the function $v(t)$ is the same function as in the Theorem 2.1. Then the Cauchy problem (2.1), (2.2) has one unique solution $u(t, r)$ in the form $u(t, r)=v(t) \omega(r)$ for which $u(t, r) \in N, u(t, r) \in \dot{H}^{1}\left(\left[r_{0}, \infty\right)\right)$ for $\forall t \in[0,1]$, and the solution map is not uniformly continuous.

Proof. In the Theorem 2.1 was proved that the equation (2.1) has one unique nontrivial solution $\tilde{u}(t, r)=v(t) \omega(r)$ for which $\tilde{u}(t, r) \in N$. Also, for every $k \in \mathcal{N}$ and for every fixed $t \in[0,1]$ we have

$$
\begin{aligned}
& \left\|\frac{\partial \tilde{u}}{\partial r}\right\|_{L^{2}\left(\left[r_{0}, \infty\right)\right)}^{2}= \\
& =\int_{r_{0}}^{\infty}\left(\frac{1}{r^{n}} \int_{r}^{\infty} s^{n}\left(\frac{v^{\prime}(t)}{v(t)} \tilde{u}-f(t, \tau, \tilde{u})\right) d s\right)^{2} d r \leq \\
& \leq \int_{r_{0}}^{\infty}\left(\frac{1}{r^{n}} \int_{r}^{\infty} s^{n}\left(\frac{v^{\prime}(t)}{v(t)} \tilde{u}+f(t, \tau, \tilde{u})\right) d s\right)^{2} d r \leq \\
& \leq \int_{r_{0}}^{\infty}\left(\frac{1}{r^{n}} \int_{r}^{\infty} s^{n}\left(A_{2}+\frac{b}{2 B}\right) \tilde{u} d s\right)^{2} d r= \\
& =\left(A_{2}+\frac{b}{2 B}\right)^{2} \int_{r_{0}}^{\infty}\left(\frac{1}{r^{n}} \int_{r}^{\infty} \sqrt{s^{2 n+2 k} \tilde{u}} \sqrt{\tilde{u}} \frac{1}{s^{k}} d s\right)^{2} d r \leq \\
& \leq\left(A_{2}+\frac{b}{2 B}\right)^{2} \int_{r_{0}}^{\infty}\left(\frac{1}{r^{n}} \int_{r}^{\infty} \sqrt{\tilde{u}} \frac{1}{s^{k}} d s\right)^{2} d r \leq \\
& \leq\left(A_{2}+\frac{b}{2 B}\right)^{2} \int_{r_{0}}^{\infty}\left(\frac{1}{r^{n}}\left(\int_{r}^{\infty} \tilde{u}^{2} d s\right)^{\frac{1}{4}}\left(\int_{r}^{\infty} \frac{1}{s^{\frac{4 k}{3}}} d s\right)^{\frac{3}{4}}\right)^{2} d r \leq \\
& \leq\left(A_{2}+\frac{b}{2 B}\right)^{2} \frac{1}{\left(\frac{4 k}{3}-1\right)^{\frac{3}{2}}\left(2 n+2 k-\frac{5}{2}\right) r_{0}^{2 n+2 k-\frac{5}{2}}}\|\tilde{u}\|_{L^{2}\left(\left[r_{0}, \infty\right)\right)<\infty}
\end{aligned}
$$

because $\tilde{u} \in L^{2}\left(\left[r_{0}, \infty\right)\right)$. Consequently $\tilde{u} \in \dot{H}^{1}\left(\left[r_{0}, \infty\right)\right)$ for every fixed $t \in[0,1]$. 
Now we suppose that the solution map $\left(u_{0}, u_{1}\right) \longrightarrow u(t, r)$ is uniformly continuous.

Let

$$
0<\epsilon<\left(A_{1}-\frac{b}{2 B}\right)^{2} \frac{1}{A^{2}}\left(d_{1}-c_{1}\right)^{3} \frac{c_{1}^{2 n}}{d_{1}^{2 n}}
$$

Let also

$$
u_{1}=\tilde{u}, \quad u_{2}=0 .
$$

Then there exists positive constant $\delta$ such that

$$
\begin{aligned}
& E\left(0, u_{1}-u_{2}\right) \leq \delta \\
& \text { and } \\
& E\left(1, u_{1}-u_{2}\right) \leq \epsilon
\end{aligned}
$$

From here

$$
\begin{aligned}
& \epsilon \geq E\left(1, u_{1}-u_{2}\right)=E(1, \tilde{u}) \geq \int_{r_{0}}^{\infty}\left(\frac{1}{r^{n}} \int_{r}^{\infty} s^{n}\left(\frac{v^{\prime}(1)}{v(1)} \tilde{u}-f(1, s, \tilde{u})\right) d s\right)^{2} d r \geq \\
& \geq \int_{c_{1}}^{d_{1}}\left(\frac{1}{r^{n}} \int_{c_{1}}^{d_{1}} s^{n}\left(\frac{v^{\prime}(1)}{v(1)} \tilde{u}-f(1 . s, \tilde{u})\right) d s\right)^{2} d r \geq \\
& \geq \int_{c_{1}}^{d_{1}}\left(\frac{1}{r^{n}} \int_{c_{1}}^{d_{1}} s^{n}\left(\frac{v^{\prime}(1)}{v(1)} \tilde{u}-\frac{b}{2 B} \tilde{u}\right) d s\right)^{2} d r \geq \\
& \geq \int_{c_{1}}^{d_{1}}\left(\frac{1}{r^{n}} \int_{c_{1}}^{d_{1}} s^{n}\left(\frac{v^{\prime}(1)}{v(1)}-\frac{b}{2 B}\right) \frac{1}{A} d s\right)^{2} d r \geq \\
& \geq\left(A_{1}-\frac{b}{2 B}\right)^{2} \frac{1}{A^{2}}\left(d_{1}-c_{1}\right)^{3} \frac{c_{1}^{2 n}}{d_{1}^{2 n}}
\end{aligned}
$$

which is a contradiction with (2.6).

\section{Appendix}

Lemma 3.1. The set $N$ is a closed subset of $\mathcal{C}\left([0,1] L^{2}\left(\left[r_{0}, \infty\right)\right)\right)$.

Proof. Let $t \in[0,1]$ is fixed.

Let also $\left\{u_{n}\right\}$ is a sequence of elements of the set $N$ for which

$$
\lim _{n \longrightarrow \infty}\left\|u_{n}-\tilde{u}\right\|_{L^{2}\left(\left[r_{0}, \infty\right)\right)}=0
$$

where $\tilde{u} \in L^{2}\left(\left[r_{0}, \infty\right)\right)$. Since $P(u)$ is a continuous- differentiable function of $u$, for $r \in\left[r_{0}, r_{0}+1\right]$ and $u \in N$ we have

$$
\begin{aligned}
& P^{\prime}(u)=\int_{r}^{\infty} \frac{1}{s^{n}} \int_{s}^{\infty} \tau^{n}\left(\frac{v^{\prime}(t)}{v(t)}-f_{u}^{\prime}(t, \tau, u)\right) d \tau d s \geq \\
& \geq \int_{r}^{\infty} \frac{1}{s^{n}} \int_{s}^{\infty} \tau^{n}\left(A_{1}-\frac{b}{B}\right) d \tau d s \geq \\
& \geq \int_{r_{0}+1}^{r_{0}+2} \frac{1}{s^{n}} \int_{r_{0}+1}^{r_{0}+2} \tau^{n}\left(A_{1}-\frac{b}{B}\right) d \tau d s \geq \\
& \geq\left(A_{1}-\frac{b}{B}\right) \frac{\left(r_{0}+1\right)^{n}}{\left(r_{0}+2\right)^{n}} .
\end{aligned}
$$

From here follows that for every $u \in N$ there exists

$$
L=\min _{r \in\left[r_{0}, r_{0}+1\right]}\left|P^{\prime}(u)(r)\right|>0 .
$$

Let

$$
M_{1}=\max _{r \in\left[r_{0}, r_{0}+1\right]}\left|\frac{\partial}{\partial r} P^{\prime}(u)(r)\right| .
$$

Now we will prove that for every $\epsilon>0$ there exists $\delta=\delta(\epsilon)>0$ such that from $|x-y|<\delta$ we have

$$
\left|u_{m}(x)-u_{m}(y)\right|<\epsilon \quad \text { for } \quad \forall m \in \mathcal{N} \text {. }
$$


We suppose that there exists $\tilde{\epsilon}>0$ such that for every $\delta>0$ there exist natural $m$ and $x, y \in\left[r_{0}, \infty\right),|x-y|<\delta$ for which $\left|u_{m}(x)-u_{m}(y)\right| \geq \tilde{\epsilon}$. We choose $\tilde{\tilde{\epsilon}}$ such that $0<\tilde{\tilde{\epsilon}}<L \tilde{\epsilon}$. We note that $P\left(u_{m}\right)(x)$ is uniformly continuous for $x \in\left[r_{0}, \infty\right)$ ( for $u \in N P(u)(r)$ is uniformly continuous function for $r \in\left[r_{0}, \infty\right.$ ) because $P(u)(r) \in \mathcal{C}\left(\left[r_{0}, \infty\right)\right)$ and as in the proof of the Theorem 2.1 we have that there exists positive constant $C$ such that $\left.\left|\frac{\partial}{\partial r} P(u)(r)\right| \leq C\right)$. Then there exists $\delta_{1}=\delta_{1}(\tilde{\tilde{\epsilon}})>0$ such that for every natural $m$ we have

$$
\left|P\left(u_{m}\right)(x)-P\left(u_{m}\right)(y)\right|<\tilde{\tilde{\epsilon}}, \quad \forall x, y \in\left[r_{0}, \infty\right):|x-y|<\delta_{1} .
$$

Consequently we can choose

$$
0<\delta<\min \left\{1, \delta_{1}, \frac{(L \tilde{\epsilon}-\tilde{\tilde{\epsilon}}) B}{M_{1}}\right\}
$$

such that there exist natural $m$ and $x_{1}, x_{2} \in\left[r_{0}, \infty\right)$ for which

$$
\left|x_{1}-x_{2}\right|<\delta, \quad\left|u_{m}\left(x_{1}-x_{2}+r_{0}\right)-u_{m}\left(r_{0}\right)\right| \geq \tilde{\epsilon}
$$

In particular

$$
\left|P\left(u_{m}\right)\left(x_{1}-x_{2}+r_{0}\right)-P\left(u_{m}\right)\left(r_{0}\right)\right|<\tilde{\tilde{\epsilon}}
$$

Let us suppose for convinience that $x_{1}-x_{2}>0$. Then $x_{1}-x_{2}<1$ and for every $u \in N$ we have $P^{\prime}(u)\left(x_{1}-x_{2}+r_{0}\right) \geq L$. Then from the middle point theorem we have

$$
\begin{aligned}
& P(0)=0, P\left(u_{m}\right)\left(x_{1}-x_{2}+r_{0}\right)=P^{\prime}(\xi)\left(x_{1}-x_{2}+r_{0}\right) u_{m}\left(x_{1}-x_{2}+r_{0}\right), \\
& P\left(u_{m}\right)\left(r_{0}\right)=P^{\prime}(\xi)\left(r_{0}\right) u_{m}\left(r_{0}\right), \\
& \left|P\left(u_{m}\right)\left(x_{1}-x_{2}+r_{0}\right)-P\left(u_{m}\right)\left(r_{0}\right)\right|= \\
& =\left|P^{\prime}(\xi)\left(x_{1}-x_{2}+r_{0}\right) u_{m}\left(x_{1}-x_{2}+r_{0}\right)-P^{\prime}(\xi)\left(r_{0}\right) u_{m}\left(r_{0}\right)\right|= \\
& =\mid P^{\prime}(\xi)\left(x_{1}-x_{2}+r_{0}\right) u_{m}\left(x_{1}-x_{2}+r_{0}\right)-P^{\prime}(\xi)\left(x_{1}-x_{2}+r_{0}\right) u_{m}\left(r_{0}\right)+ \\
& +P^{\prime}(\xi)\left(x_{1}-x_{2}+r_{0}\right) u_{m}\left(r_{0}\right)-P^{\prime}(\xi)\left(r_{0}\right) u_{m}\left(r_{0}\right) \mid \geq \\
& \geq\left|P^{\prime}(\xi)\left(x_{1}-x_{2}+r_{0}\right) u_{m}\left(x_{1}-x_{2}+r_{0}\right)-P^{\prime}(\xi)\left(x_{1}-x_{2}+r_{0}\right) u_{m}\left(r_{0}\right)\right|- \\
& -\left|P^{\prime}(\xi)\left(x_{1}-x_{2}+r_{0}\right) u_{m}\left(r_{0}\right)-P^{\prime}(\xi)\left(r_{0}\right) u_{m}\left(r_{0}\right)\right|= \\
& =\left|P^{\prime}(\xi)\left(x_{1}-x_{2}+r_{0}\right) u_{m}\left(x_{1}-x_{2}+r_{0}\right)-P^{\prime}(\xi)\left(x_{1}-x_{2}+r_{0}\right) u_{m}\left(r_{0}\right)\right|- \\
& -\left|\frac{\partial}{\partial r} P^{\prime}(\xi)\right|\left|x_{1}-x_{2}\right|\left|u_{m}\left(r_{0}\right)\right| \geq \\
& \geq L \tilde{\epsilon}-M_{1} \delta \frac{1}{B} \geq \tilde{\tilde{\epsilon}},
\end{aligned}
$$

which is a contradiction with (3.1). Therefore, for every $\epsilon>0$ there exists $\delta=$ $\delta(\epsilon)>0$ such that from $|x-y|<\delta$ follows

$$
\left|u_{m}(x)-u_{m}(y)\right|<\epsilon \quad \forall m \in \mathcal{N} .
$$

On the other hand from the definition of the set $N$ we have that for every natural m

$$
u_{m}(r) \leq \frac{1}{B} \quad \forall r \geq r_{0}
$$

From (3.2) and (3.3) follows that the set $\left\{u_{m}\right\}$ is a compact subset of the space $\mathcal{C}\left(\left[r_{0}, \infty\right)\right)$. Therefore there is a subsequence $\left\{u_{n_{k}}\right\}$ and function $u \in \mathcal{C}\left(\left[r_{0}, \infty\right)\right)$ for which

$$
\left|u_{n_{k}}(x)-u(x)\right|<\epsilon \quad \forall x \in\left[r_{0}, \infty\right) .
$$


Now we suppose that there is no true that $u=\tilde{u}$ a.e. in $\left[r_{0}, \infty\right)$. Then there exist $\epsilon_{1}>0$ and subinterval $\Delta \subset\left[r_{0}, \infty\right)$ such that $\mu(\Delta)>0$ and

$$
|u-\tilde{u}|>\epsilon_{1} \quad \text { for } \quad r \in \Delta \text {. }
$$

Let $\epsilon>0$ is chosen such that

$$
\epsilon<\frac{\epsilon_{1}(\mu(\Delta))^{\frac{1}{2}}}{\mu(\Delta)^{\frac{1}{2}}+1} .
$$

Then, for every enough large $n_{k} \in \mathcal{N}$, we have

$$
\begin{aligned}
& \left\|u_{n_{k}}-\tilde{u}\right\|_{L^{2}\left(\left[r_{0}, \infty\right)\right)}<\epsilon, \\
& \epsilon \mu(\Delta)=\epsilon \int_{\Delta} d x>\int_{\Delta}\left|u_{n_{k}}-u\right| d x= \\
& =\int_{\Delta}\left|u_{n_{k}}-\tilde{u}+\tilde{u}-u\right| d x \geq \\
& \geq \int_{\Delta}|\tilde{u}-u| d x-\int_{\Delta}\left|u_{n_{k}}-\tilde{u}\right| d x \geq \\
& \geq \epsilon_{1} \mu(\Delta)-\left(\int_{\Delta}\left|u_{n_{k}}-\tilde{u}\right|^{2} d x\right)^{\frac{1}{2}}(\mu(\Delta))^{\frac{1}{2}} \geq \\
& \geq \epsilon_{1} \mu(\Delta)-\left\|u_{n_{k}}-\tilde{u}\right\|_{L^{2}\left(\left[r_{0}, \infty\right)\right)}(\mu(\Delta))^{\frac{1}{2}}> \\
& >\epsilon_{1} \mu(\Delta)-\epsilon(\mu(\Delta))^{\frac{1}{2}},
\end{aligned}
$$

which is a contradiction with (3.4). From here $u=\tilde{u}$ a.e. in $\left[r_{0}, \infty\right),\left|u_{n}-u\right|^{2}=$ $\left|\tilde{u}-u_{n}\right|^{2}$ a.e. in $\left[r_{0}, \infty\right),\left\|u_{n}-u\right\|_{L^{2}\left(\left[r_{0}, \infty\right)\right)}=\left\|u_{n}-\tilde{u}\right\|_{L^{2}\left(\left[r_{0}, \infty\right)\right)}$.

Consequently, for every sequence $\left\{u_{n}\right\}$ from elements of the set $N$, which is convergent in $L^{2}\left(\left[r_{0}, \infty\right)\right)$, there exists a function $u \in \mathcal{C}\left(\left[r_{0}, \infty\right)\right), u \in L^{2}\left(\left[r_{0}, \infty\right)\right)$ for which

$$
\lim _{n \longrightarrow \infty}\left\|u_{n}-u\right\|_{L^{2}\left(\left[r_{0}, \infty\right)\right)}=0 .
$$

Bellow we will suppose that $\left\{u_{n}\right\}$ is a sequence from elements of the set $N$, which is convergent in $L^{2}\left(\left[r_{0}, \infty\right)\right)$. Then there exists a function $u \in \mathcal{C}\left(\left[r_{0}, \infty\right)\right)$, $u \in L^{2}\left(\left[r_{0}, \infty\right)\right)$ for which

$$
\lim _{n \longrightarrow \infty}\left\|u_{n}-u\right\|_{L^{2}\left(\left[r_{0}, \infty\right)\right)}=0 .
$$

Now we suppose that $u(t, \infty) \neq 0$. Then there exist enough large $Q>0$, enough large natural $m$ and $\epsilon_{2}>0$ for which

$$
u_{m}(t, r)=0, \quad u(t, r)>\epsilon_{2}, \quad \forall r \geq Q .
$$

We choose

$$
0<\epsilon_{3}<\epsilon_{2}
$$

Then, for every enough large $n \in \mathcal{N}$ we have $\left|u_{n}(t, r)-u(t, r)\right|<\epsilon_{3}$ and

$$
\begin{aligned}
& \epsilon_{3}>\int_{Q}^{Q+1}\left|u_{n}(t, r)-u(t, r)\right| d r \geq \\
& \geq \int_{Q}^{Q+1}\left(|u(t, r)|-\left|u_{n}(t, r)\right|\right) d r= \\
& =\int_{Q}^{Q+1}|u(t, r)| d r>\epsilon_{2},
\end{aligned}
$$

which is a contradiction with (3.5). Therefore $u(t, \infty)=0$.

Now we will prove that $\frac{\partial}{\partial t} u(t, r)$ exists for every $t \in[0,1]$. Let us suppose that $r \in\left[r_{0}, \infty\right)$ is fixed and there exists $t_{1} \in[0,1]$ such that $\frac{\partial}{\partial t} u\left(t_{1}, r\right)$ no exists. Then for every $h>0$, which is enough small, exists $\epsilon_{4}>0$ such that

$$
\left|\frac{u\left(t_{1}+h, r\right)-u\left(t_{1}, r\right)}{h}\right|>\epsilon_{4}
$$


and

$$
0<\epsilon_{5}<\frac{h}{2} \epsilon_{4},
$$

such that

$$
\left|u_{n}\left(t_{1}+h, r\right)-u\left(t_{1}, r\right)\right|<\epsilon_{5} .
$$

From here

$$
\begin{aligned}
& \epsilon_{5}>\left|u_{n}\left(t_{1}+h, r\right)-u\left(t_{1}+h, r\right)\right|= \\
& =\left|u_{n}\left(t_{1}+h, r\right)-u\left(t_{1}, r\right)+u\left(t_{1}, r\right)-u\left(t_{1}+h, r\right)\right| \geq \\
& \geq\left|u\left(t_{1}, r\right)-u\left(t_{1}+h, r\right)\right| \frac{1}{h} h-\left|u_{n}\left(t_{1}+h, r\right)-u\left(t_{1}, r\right)\right| \geq \epsilon_{4} h-\epsilon_{5},
\end{aligned}
$$

which is a contradiction of our choice of $\epsilon_{5}$. Therefore $\frac{\partial}{\partial t} u(t, r)$ exists for every $t \in[0,1]$. As in above we can see that $u(t, r) \in \mathcal{C}^{1}([0,1])$ for every $r \geq r_{0}, u(t, r) \in$ $\mathcal{C}^{2}\left(\left[r_{0}, \infty\right)\right)$ for every $t \in[0,1], u_{r}(t, \infty)=0$ for every $t \in[0,1]$.

Now we suppose that there exists interval $\Delta_{2} \subset\left[r_{0}, \infty\right)$ such that

$$
u(t, r) \geq \frac{1}{B}+\epsilon_{7} \quad \text { for } \quad r \in \Delta_{2} .
$$

Let $n \in \mathcal{N}$ is enough large and $\epsilon_{8}>0$ are chosen such that

$$
\left|u_{n}(t, r)-u(t, r)\right|<\epsilon_{8} \quad \text { for } \quad r \in \Delta_{2}, 0<\epsilon_{8}<\epsilon_{7} .
$$

From here, for $r \in \Delta_{2}$ we have

$$
\epsilon_{8}>\left|u_{n}(t, r)-u(t, r)\right| \geq|u(t, r)|-\left|u_{n}(t, r)\right| \geq \frac{1}{B}+\epsilon_{7}-\frac{1}{B}=\epsilon_{7},
$$

which is one contradiction with (3.7). Therefore we have $u(t, r) \leq \frac{1}{B}$ for every $r \geq r_{0}$.

Now we suppose that there exists interval $\Delta_{3} \subset\left[c_{1}, d_{1}\right]$ for which $u(t, r)<\frac{1}{A}$ for every $r \in \Delta_{3}$. From here there exists $\epsilon_{9}>0$ such that $u(t, r) \leq \frac{1}{A}-\epsilon_{9}$ for $r \in \Delta_{3}$. Also, let

$$
0<\epsilon_{10}<\epsilon_{9}
$$

and $n \in \mathcal{N}$ is enough large such that $\epsilon_{10}>\left|u_{n}(t, r)-u(t, r)\right|$ for $r \in \Delta_{3}$. Then for $r \in \Delta_{3}$ we have

$$
\epsilon_{10}>\left|u_{n}(t, r)-u(t, r)\right| \geq\left|u_{n}(t, r)\right|-|u(t, r)| \geq \frac{1}{A}-\frac{1}{A}+\epsilon_{9},
$$

which is one contradiction with (3.8). Consequently, for every $r \in\left[c_{1}, d_{1}\right]$ we have $u(t, r) \geq \frac{1}{A}$.

Now we suppose that there exist $\alpha \in \mathcal{N} \cup\{0\}$, interval $\Delta_{4} \subset\left[r_{0}, \infty\right)$ and $\epsilon_{11}>0$ such that

$$
\left|r^{\alpha} u(t, r)\right|>1+\epsilon_{11} \quad \text { for } \quad r \in \Delta_{4} .
$$

Let $\epsilon_{12}>0$ and $n \in \mathcal{N}$ are chosen such that

$$
\left|r^{\alpha}\left(u_{n}(t, r)-u(t, r)\right)\right|<\epsilon_{12} \quad \text { for } \quad r \in \Delta_{4}, \quad 0<\epsilon_{12}<\epsilon_{11} .
$$

From here

$$
\epsilon_{12}>\left|r^{\alpha}\left(u_{n}(t, r)-u(t, r)\right)\right| \geq\left|r^{\alpha} u(t, r)\right|-r^{\alpha}\left|u_{n}(t, r)\right| \geq \epsilon_{11},
$$

which is a contradiction with (3.9). Therefore for every $\alpha \in \mathcal{N} \cup\{0\}$ and for every $r \in\left[r_{0}, \infty\right)$ we have $r^{\alpha} u(t, r) \leq 1$. After we use the same arguments we can see that for every $\alpha \in \mathcal{N} \cup\{0\}$ and for every $r \in\left[r_{0}, \infty\right)$ we have $r^{\alpha}\left|u_{r}(t, r)\right| \leq 1$. 
Now we suppose that there exist interval $\Delta_{5} \subset\left[r_{0}, \infty\right)$ and $\epsilon_{13}>0$ such that for $r \in \Delta_{5}$ we have

$$
u(t, r)<-\epsilon_{13} .
$$

Let $n \in \mathcal{N}$ is enough large and $\epsilon_{14}>0$ are fixed for which

$$
\left|u_{n}(t, r)-u(t, r)\right|<\epsilon_{14} \quad \text { for } \quad r \in \Delta_{5}, \quad 0<\epsilon_{14}<\epsilon_{13} .
$$

Then for $r \in \Delta_{5}$ we have

$$
\epsilon_{14}>u_{n}(t, r)-u(t, r)>\epsilon_{13}
$$

which is one contradiction with (3.10).

\section{References}

[1] Georgiev, S. Blow up of solutions for Klein-Gordon equations in the Reissner-Nordström metric, EJDE, Vol 2005(2005), No 67, pp. 1-22.

[2] Georgiev, S. Blow - up of the solutions of nonlinear wave equation in Reissner - Nordström metric, Dynamics of PDE, Vol. 3, No. 4, 295-329, 2006.

[3] Georgiev, S. Uniform continuity of the solution map for nonlinear wave equation, EJQTDE, Vol 2007( 2007), No 12, pp. 1-14.

[4] Georgiev, S. Blow - up of the solutions of nonlinear wave equation, BVP, 2007, Q2.

[5] Krasnosel'skii, M., P.Zabrejko, Geometrical Methods of Nonlinear Analysis, Nauka, Moscow, 1975. /In Russian/

Department of Differential Equations, University of Sofia, Sofia 1164, Bulgaria E-mail address: sgg2000bg@yahoo.com 